This item was submitted to Loughborough's Research Repository by the author.

Items in Figshare are protected by copyright, with all rights reserved, unless otherwise indicated.

\title{
A generalization of Ramsey rule on discount rate with regime switching
}

PLEASE CITE THE PUBLISHED VERSION

https://doi.org/10.1016/j.econlet.2018.06.011

PUBLISHER

Elsevier

VERSION

AM (Accepted Manuscript)

PUBLISHER STATEMENT

This paper was accepted for publication in the journal Economics Letters and the definitive published version is available at https://doi.org/10.1016/j.econlet.2018.06.011

\section{LICENCE}

CC BY-NC-ND 4.0

\section{REPOSITORY RECORD}

Park, Seyoung. 2019. "A Generalization of Ramsey Rule on Discount Rate with Regime Switching”. figshare. https://hdl.handle.net/2134/33773. 


\section{Accepted Manuscript}

A generalization of Ramsey rule on discount rate with regime switching

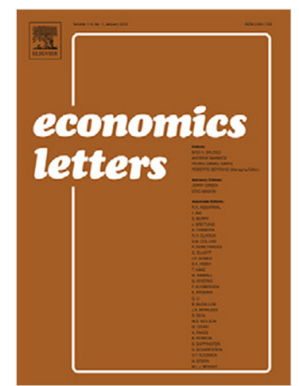

PII:

S0165-1765(18)30234-9

DOI: $\quad$ https://doi.org/10.1016/j.econlet.2018.06.011

Reference: $\quad$ ECOLET 8084

To appear in: $\quad$ Economics Letters

Received date: 14 March 2018

Revised date: 8 June 2018

Accepted date : 12 June 2018

Please cite this article as: Park S., A generalization of Ramsey rule on discount rate with regime switching. Economics Letters (2018), https://doi.org/10.1016/j.econlet.2018.06.011

This is a PDF file of an unedited manuscript that has been accepted for publication. As a service to our customers we are providing this early version of the manuscript. The manuscript will undergo copyediting, typesetting, and review of the resulting proof before it is published in its final form. Please note that during the production process errors may be discovered which could affect the content, and all legal disclaimers that apply to the journal pertain. 


\section{Highlights}

- The Ramsey rule on the discount rate is extended to regime-dependent interest-rate formulas.

- The effect of the regime switching is dominant.

- Dynamic programming is used to derive the generalized equilibrium discount rate in closed form. 


\title{
A Generalization of Ramsey Rule on Discount Rate with Regime Switching*
}

Seyoung Park ${ }^{\dagger}$

\begin{abstract}
I generalize the following rule of Ramsey (1928) on the discount rate with regime switching: the discount rate is the sum of the rate of pure time preference and the product of the consumption elasticity of marginal utility and the consumption growth rate. The Ramsey rule can be extended to regime-dependent interest-rate formulas for discounting future regime changes. Notwithstanding debate about empirically plausible values of the rate of pure time preference, I theoretically show that the effect of pure time preference is overwhelmingly dominated by the effect of the regime switching parameter. This is closely associated with consumption smoothing consequences across regimes.
\end{abstract}

Keywords: discount rate, regime switching, equilibrium

JEL Classifications: C61, D52, G11, G12

*This research was supported by a grant from IREC, The Institute of Finance and Banking, Seoul National University for which the author is indebted. The author is also grateful for the helpful discussions with Li Haitao, Min Dai, Steven Kou, Huainan Zhao, and Andrew Vivian. All errors are the author's own responsibility.

${ }^{\dagger}$ School of Business and Economics, Loughborough University, Tel: +44-07927-494518, E-mail: 


\section{Introduction}

Since the seminal paper of Ramsey (1928), economists have assumed that agents are impatient. This can be modelled as agents who obtain their future utility at a discount from current utility. This has been applied to neoclassical economists' expected utility theory. The bottom line is that agents' lifetime utility is comprised of the sum of discounted flows of current and future utilities, thereby the discount is captured by the rate of pure time preference. However, it has been a challenge to get economists to shake hands on an appropriately agreed rate of pure time preference. In line with this, Ramsey (1928) claims

One point should perhaps be emphasized more particularly; it is assumed that we do not discount later enjoyments in comparison with earlier ones, a practice which is ethically indefensible and arises merely from the weakness of the imagination.

This is precisely the direction I would like to explore here. I generalize the following rule of Ramsey (1928) on the discount rate with regime switching: ${ }^{1}$ the discount rate is the sum of the rate of pure time preference and the product of

\footnotetext{
${ }^{1}$ In the aftermath of the global economic crisis of 2008, regime switching has attracted much interest recently to appropriately account for business cycle expansions and recessions. As John H. Cochrane (2017) arguably states in the introduction of his paper,

Asset prices and returns are correlated with business cycles. Stocks rise in good times, and fall in bad times. Real and nominal interest rates rise and fall with the business cycle. Stock returns and bond yield also help to forecast macroeconomic events such as GDP growth and inflation.

In light of the stylized behavior of economic cycles in the long run, regime switching models have become standard elements in economic modelling. Theoretical and empirical advances, depending on and extending Hamilton's (1989) seminal application of regime switching to economic recessions and expansions, have easily permitted the identification of stylized behavior of data such as time-varying properties.
} 
the consumption elasticity of marginal utility and the consumption growth rate. ${ }^{2}$ For analytical crispness, I assume that there are two regimes ("Bull" and "bear") with different fundamental parameters such as the expected rate and volatility of consumption growth. The Ramsey rule can be extended to regime-dependent interest-rate formulas for discounting future regime changes. The generalizedRamsey formula for the discount rate with regime switching shows that the effect of pure time preference is overwhelmingly dominated by the effect of the regime switching parameter. This is closely associated with consumption smoothing consequences across regimes. Unlike the case without regime switching, agents are willing to achieve cross-regime consumption smoothing, which is reflected in the generalized-Ramsey formula.

\section{The Model}

I consider an infinite-horizon economy with a single consumption good (the numeraire). Uncertainty is driven by a filtered probability space $\left(\Omega, \mathcal{F},\left\{\mathcal{F}_{t}\right\}, P\right)$ in which a multi-dimensional Brownian motion and one Poisson process representing regime-switching risk are defined. All stochastic processes are adapted to $\left\{\mathcal{F}_{t}\right\}$, which is the $P$-augmentation of the filtration generated by the Brownian motion and Poisson process. All stated stochastic processes are assumed to be well defined, without explicitly stating the regularity conditions ensuring this.

Investment opportunities are represented by the financial market comprised of one instantaneously riskless bond in zero net supply, and multiple stocks each in

\footnotetext{
${ }^{2}$ This well-known Ramsey rule on the discount rate has served as a key role in an optimal intertemporal allocation when dealing with the productivity of capital or fundamentally, the return on investment.
} 
constant net supply of 1 and paying dividends. The fundamental parameters in the investment opportunities are regime dependent. There are two regimes, "(B)ull" (regime $B$ ) and "(b)ear" (regime $b$ ). Regime $i$ switches into regime $j$ at the first time of an independent Poisson process with intensity $\delta_{i}$, for $i, j \in\{B, b\}$. In regime $i$, the bond price $B$ and the stock prices $S$ are given by

$$
d B(t)=r_{i} B(t) d t
$$

and

$$
d S(t)+D(t) d t=S(t)\left\{\mu_{i} d t+\sigma_{i}^{\top} d Z(t)\right\}
$$

where $r_{i}$ is the constant risk-free interest rate, $D(t)$ is the dividend vector process, $\mu_{i}$ is the constant mean vector, $\sigma_{i}$ is the constant nonsingular standard deviation matrix, and $Z(t)$ is the Brownian motion with dimensionality equal to the number of stocks.

For the given adapted nonnegative consumption process $c(t)$ and adapted portfolio process $\pi(t)$, an agent accumulates her wealth $W(t)$ according to the following dynamic budget constraint: in regime $i$,

$d W(t)=\left\{r_{i} W(t)-c(t)+\pi(t)^{\top}\left(\mu_{i}-r_{i} \mathbf{1}\right)\right\} d t+\pi(t)^{\top} \sigma_{i}^{\top} d Z(t), W(0)=w \geq 0$,

which is subject to the solvency constraint $W(t) \geq 0$, where $\mathbf{1}$ is a vector of 1 's with dimensionality equal to the number of stocks. I denote by $\mathcal{A}(w)$ the set of admissible policies of consumption process $c(t)$ and portfolio process $\pi(t)$ such that the dynamic budget constraint stated above is satisfied.

Each representative agent is assumed to derive utility from consumption in the 
form of

$$
\int_{0}^{\infty} e^{-\beta t} U(c(t)) d t
$$

where $\beta>0$ is the rate of pure time preference (or equivalently, the rate of impatience), and $U(\cdot)$ measures the agent's utility and is twice continuously differentiable, strictly increasing, and strictly concave. Motivated by neoclassical economists' expected utility theory, the representative agent's optimization problem is described by finding the value function as follows: in regime $i$,

$$
V_{i}(w) \equiv \sup _{(c, \pi) \in \mathcal{A}(w)} E\left[\int_{0}^{\infty} e^{-\beta t} U(c(t)) d t\right]
$$

subject to regime switching with different fundamental parameters in the investment opportunities such as the expected rate and volatility of stock returns. The agent aims to maximize her expected utility from consumption by optimally controlling per-period consumption process $c(t)$ and portfolio process $\pi(t)$ that are regime dependent. After integrating out the Poisson process representing the regime-switching risk, the agent solves

$$
V_{i}(w)=\sup _{(c, \pi) \in \mathcal{A}(w)} E\left[\int_{0}^{\infty} e^{-\left(\beta+\delta_{i}\right) t}\left\{U(c(t))+\delta_{i} V_{j}(W(t))\right\} d t\right]
$$

\section{A Generalized-Ramsey Formula for Discount Rate}

I examine a simple exchange economy in the style of Lucas (1978). The extension is that a representative agent faces regime-shift risk. The agent receives an endowment to be consumed in equilibrium and is assumed to trade a riskless asset and multiple risky assets entitling the owner to the dividend in the economy. The 
returns to these assets adjust to represent a no-trade equilibrium. The primitives of the equilibrium model are as follows. I simply assume that in regime $i \in\{B, b\}$, the exogenously given aggregate consumption process follows a geometric Brownian motion and it is given by

$$
d D(t)=D(t)\left\{\mu_{i}^{D} d t+\left(\sigma_{i}^{D}\right)^{\top} d Z(t)\right\}
$$

where the expected instantaneous growth rate $\mu_{i}^{D}$ is the constant mean and the instantaneous volatility of growth rate $\sigma_{i}^{D}$ is the constant standard deviation vector that may be regime dependent.

Definition 3.1. An equilibrium is a collection of $\left(r_{i}, \mu_{i}, \sigma_{i}\right)$ and optimal policies $(c(t), \pi(t))$ such that the consumption good, stock, and bond markets clear, in other words,

$$
\begin{aligned}
& c(t)=D(t), \\
& \pi^{j}(t)=S^{j}(t), \quad j=1, \ldots, N, \\
& W(t)=\sum_{j=1}^{N} S^{j}(t),
\end{aligned}
$$

where $N$ is the number of multiple stocks.

Now I derive a generalized-Ramsey formula for the discount rate under the widely adoped constant relative risk aversion (CRRA) utility function:

$$
U(c(t))=\frac{c(t)^{1-\gamma}}{1-\gamma}
$$

where $\gamma>0$ is the constant coefficient of relative risk aversion.

Theorem 3.1. Under the CRRA utility function, the Ramsey rule on the discount 
rate can be generalized by the following formula: for $i, j \in\{B, b\}, i \neq j$, regimedependent discount rates are given by

$$
r_{i}=\beta+\gamma \mu_{i}^{D}-\frac{1}{2} \gamma(1+\gamma)\left\|\sigma_{i}^{D}\right\|^{2}+\delta_{i}\left\{1-\frac{M_{j}\left(r_{j}\right)}{M_{i}\left(r_{i}\right)}\right\}
$$

where $M_{i}\left(r_{i}\right)$ and $M_{j}\left(r_{j}\right)$ satisfy the following system of algebraic equations:

$$
-\left(\eta_{i}\left(r_{i}\right)+\delta_{i}\right) M_{i}\left(r_{i}\right)+\gamma M_{i}\left(r_{i}\right)^{1-1 / \gamma}+\delta_{i} M_{j}\left(r_{j}\right)=0,
$$

where

$$
\eta_{i}\left(r_{i}\right)=\beta-(1-\gamma)\left(r_{i}+\frac{\left\|\theta_{i}\right\|^{2}}{2 \gamma}\right), \theta_{i}=\gamma \sigma_{i}^{D}
$$

Proof. The proof of the theorem is carried out through three steps.

Step 1. In the first step, I solve the optimization problem formulated by (2) using dynamic programming. The value function $V_{i}(w)$ should satisfy the Hamilton-Jacobi-Bellman (HJB) equation, omitting time arguments to simplify notation (Jang et al., 2007): ${ }^{3}$

$$
\begin{aligned}
\sup _{(c, \pi)}\left\{\frac{1}{2}\left(\pi^{\top} \sigma_{i}\right)\left(\pi^{\top} \sigma_{i}\right)^{\top} V_{i w w}+r_{i} w V_{i w}\right. & -c V_{i w}+\pi^{\top}\left(\mu_{i}-r_{i} \mathbf{1}\right) V_{i w} \\
& \left.-\left(\beta+\delta_{i}\right) V_{i}+\delta_{i} V_{j}+\frac{c^{1-\gamma}}{1-\gamma}\right\}=0
\end{aligned}
$$

where $i, j \in\{B, b\}, i \neq j, V_{i x}$ denotes partial derivatives of value function $V_{i}$ with respect to $x$. By solving the HJB equation (5) with the first-order conditions of consumption $c$ and portfolio $\pi$, the value function $V_{i}(w)$ in regime $i \in\{B, b\}$

${ }^{3}$ When considering one riskless bond and one risky stock, the HJB equation reduces to that of Jang et al. (2007) in the absence of transaction costs. 
is obtained in closed form:

$$
V_{i}(w)=M_{i} \frac{w^{1-\gamma}}{1-\gamma}
$$

where $M_{i}$ is a regime-dependent constant, satisfying the system of algebraic equations:

$$
\begin{gathered}
-\left(\eta_{i}+\delta_{i}\right) M_{i}+\gamma M_{i}^{1-1 / \gamma}+\delta_{i} M_{j}=0 \\
\eta_{i}=\beta-(1-\gamma)\left(r_{i}+\frac{\left\|\theta_{i}\right\|^{2}}{2 \gamma}\right), \quad \theta_{i}=\left(\sigma_{i}^{\top}\right)^{-1}\left(\mu_{i}-r_{i} \mathbf{1}\right), \quad i, j \in\{B, b\}, \quad i \neq j .
\end{gathered}
$$

Accordingly, the optimal consumption and portfolio processes in regime $i \in$ $\{B, b\}$ are also obtained in closed form:

$$
c(t)=M_{i}^{-1 / \gamma} W(t) \text { and } \pi(t)=\frac{1}{\gamma}\left(\sigma_{i}\right)^{-1} \theta_{i} W(t) .
$$

Step 2. In the second step, I derive the general equilibrium risk premium. Recall the dynamic budget constraint given in (1):

$$
d W(t)=\left\{r_{i} W(t)-c(t)+\pi(t)^{\top}\left(\mu_{i}-r_{i} \mathbf{1}\right)\right\} d t+\pi(t)^{\top} \sigma_{i}^{\top} d Z(t) .
$$

Substituting the optimal consumption and portfolio processes given in (7) for $c(t)$ and $\pi(t)$ in the above budget constraint gives rise to

$$
d W(t)=W(t)\left[\left\{r_{i}-M_{i}^{-1 / \gamma}+\frac{1}{\gamma}\left\|\theta_{i}\right\|^{2}\right\} d t+\frac{1}{\gamma} \theta_{i}^{\top} d Z(t)\right]
$$


Note that using the optimal portfolio process given in (7)

$$
\sum_{j=1}^{N} S^{j}(t)=\sum_{j=1}^{N} \pi^{j}(t)=\sum_{j=1}^{N} \frac{\pi^{j}(t)}{W(t)} W(t)=\sum_{j=1}^{N} \frac{1}{\gamma}\left(\sigma_{i}\right)^{-1} \theta_{i} W(t)=W(t)
$$

the first equality comes from the clearing condition of the stock and bond markets, and the last equality also comes from the equilibrium condition that the aggregate optimal fraction invested in the risky stocks should be one. Hence, in equilibrium, the wealth $W(t)$ should be equal to the sum of the risky stock prices and the aggregate optimally invested wealth in the risky stocks:

$$
W(t)=\sum_{j=1}^{N} S^{j}(t)=\sum_{j=1}^{N} \pi^{j}(t)
$$

The equilibrium wealth dynamics can be represented by

$$
d W(t)+D(t) d t=W(t)\left\{\mu_{i}^{e m} d t+\left(\sigma_{i}^{e m}\right)^{\top} d Z(t)\right\}
$$

where $\mu_{i}^{e m}$ is the equilibrium stock market drift, $\left\|\sigma_{i}^{e m}\right\|=\sqrt{\sum_{j=1}^{N}\left(\sigma_{i j}^{e m}\right)^{2}}$ is the equilibrium stock market volatility, and these two equilibrium quantities are to be determined.

On account of the clearing condition of the consumption good,

$$
c(t)=D(t)
$$


the equilibrium wealth dynamics (9) can be rewritten as

$$
\begin{aligned}
d W(t) & =-c(t) d t+W(t)\left\{\mu_{i}^{e m} d t+\left(\sigma_{i}^{e m}\right)^{\top} d Z(t)\right\} \\
& =W(t)\left\{\left(\mu_{i}^{e m}-M_{i}^{-1 / \gamma}\right) d t+\left(\sigma_{i}^{e m}\right)^{\top} d Z(t)\right\}
\end{aligned}
$$

where the second equality is derived from the optimal consumption process given in (7). Equating (8) and (10) results in

$$
\mu_{i}^{e m}=r_{i}+\gamma\left\|\sigma_{i}^{e m}\right\|^{2}
$$

with

$$
\theta_{i}=\gamma \sigma_{i}^{e m}
$$

The equilibrium risk premium in regime $i \in\{B, b\}$ is thus:

$$
\mu_{i}^{e m}-r_{i}=\gamma\left\|\sigma_{i}^{e m}\right\|^{2}
$$

Step 3. In the final step, I derive regime-dependent discount rates $r_{i}$, generalizing the Ramsey rule on the discount rate. From the optimal consumption process given in (7), the consumption dynamics are given by

$$
\begin{aligned}
d C(t) & =M_{i}^{-1 / \gamma} d W(t) \\
& =M_{i}^{-1 / \gamma} W(t)\left[\left\{r_{i}-M_{i}^{-1 / \gamma}+\frac{1}{\gamma}\left\|\theta_{i}\right\|^{2}\right\} d t+\frac{1}{\gamma} \theta_{i}^{\top} d Z(t)\right] \\
& =M_{i}^{-1 / \gamma} W(t)\left[\left\{r_{i}-M_{i}^{-1 / \gamma}+\gamma\left\|\sigma_{i}^{e m}\right\|^{2}\right\} d t+\left(\sigma_{i}^{e m}\right)^{\top} d Z(t)\right],
\end{aligned}
$$

where the second equality is derived from the wealth dynamics (8) and the last equality comes from the equilibrium quantity (11). Recollect the aggregate con- 
sumption process given in (3):

$$
d D(t)=D(t)\left\{\mu_{i}^{D} d t+\left(\sigma_{i}^{D}\right)^{\top} d Z(t)\right\}
$$

By equating each term of $d t$ and $d Z(t)$ in (12) and (13), I obtain the following relationships:

$$
\mu_{i}^{D}=r_{i}-M_{i}^{-1 / \gamma}+\gamma\left(\sigma_{i}^{e m}\right)^{2} \text { and } \sigma_{i}^{D}=\sigma_{i}^{e m} .
$$

A little rearrangement of (6) leads to

$$
-M_{i}^{-1 / \gamma}=-\frac{\beta}{\gamma}+\frac{r_{i}}{\gamma}-r_{i}+\frac{1-\gamma}{2 \gamma^{2}}\left\|\theta_{i}\right\|^{2}+\frac{\delta_{i}}{\gamma}\left\{\frac{M_{j}}{M_{i}}-1\right\}
$$

By substituting the above equation for $-M_{i}^{-1 / \gamma}$ in (14), I complete the proof. Q.E.D.

Equation (4) simplifies to the well-known Ramsey rule on the discount rate for the special case, where there is no uncertainty over consumption growth, $\sigma_{i}^{D}=0$, without regime switching, $\delta_{i}=0$. In other words, in my equation's deterministic form the Ramsey rule demonstrates that the discount rate, $r_{i}$, is the sum of the rate of pure time preference, $\beta$, and the product of the consumption elasticity of marginal utility, $\gamma$, and the consumption growth rate, $\mu_{i}^{D}$.

The Ramsey rule can be extended to regime-dependent interest-rate formulas for discounting future regime changes. Choosing empirically plausible values of the rate of pure time preference is the ongoing debate amongst economists, but the generalized Ramsey formula for the discount rate suggested by (4) can show that the effect of pure time preference, $\beta$, is generally dominated by the effect of 
the regime switching parameter, $\delta_{i}$. More precisely, the additional term captured by $\delta_{i}\left\{1-\frac{M_{j}\left(r_{j}\right)}{M_{i}\left(r_{i}\right)}\right\}$ quantifies the regime risk premium, which is the maximum expected return an agent is willing to exchange for no regime risk. It is quite reasonable, and even numerically plausible, that agents under current regime $B$ (b) are trying to save more (less) in order to prepare for possible regime changes, as a result, the generalized discount rate is expected to decrease (increase), as reflected in the fact that the regime risk premium is negative (positive). This is closely related to consumption smoothing consequences across regimes. In the canonical Ramsey rule, agents are myopic in the sense that their consumption depends on the current regime parameters, whereas in the generalized Ramsey rule with regime switching, agents are no longer myopic and willing to achieve cross-regime consumption smoothing, considering future regime-switching times.

I provide concrete numerical examples demonstrating significant effects of regime switching stated above on the general equilibrium discount rates. I use the following baseline parameter values (Yang, 2011):

(Preferences Parameters) $\beta=0.02, \gamma=3$

(Fundamental Parameters) $\mu_{B}^{D}=0.0218, \sigma_{B}^{D}=0.0117, \mu_{b}^{D}=0.0040, \sigma_{b}^{D}=0.0387$ (Regime Intensities) $\lambda_{B}=0.42, \lambda_{b}=2.112$

Table 1 shows how the generalized regime-dependent discount rate $r_{i}$ changes with parameter values. The table also displays the discount rate averaged across 
regimes (Jang et al., 2007): ${ }^{4}$

$$
\bar{r}=\frac{\lambda_{b}}{\lambda_{B}+\lambda_{b}} r_{B}+\frac{\lambda_{B}}{\lambda_{B}+\lambda_{b}} r_{b}
$$

If $\beta$ increases by $20 \%$ of the baseline parameter value 0.02 , then the equilibrium discount rate increases from $7.18 \%$ to $7.58 \%$, i.e., an increase by $5.57 \%$ of the original level. However, the effect of changes in pure time preference $\beta$ is mitigated or strengthened after controlling for the regime switching. In particular, with increases of $\beta$ and $\lambda_{B}$ by $20 \%$ of their baseline values, the discount rate averaged across regimes increases from $7.52 \%$ to $7.76 \%$, an increase equal to $3.19 \%$ of the original average value $7.52 \%$. The increase is smaller relative to the single regime case. With increases of $\beta$ and $\lambda_{b}$ by $20 \%$ of their baseline values, the discount rate averaged across regimes increases from $7.52 \%$ to $8.04 \%$, an increase equal to $6.91 \%$ of the original average value $7.52 \%$. The increase is larger compared to the single regime case.

This gives two important implications. Firstly, misestimating the parameter values associated with regime switching or ignoring the regime switching environment can lead to misleading interpretation of discount rate in empirical analysis. Secondly, the effect of changes in values of the regime switching parameters is asymmetric and changes in the values of the bear regime parameters have a relatively greater impact on the discount rate than those in the Bull regime parameters.

\footnotetext{
${ }^{4}$ The average quantity relies on Jang et al. (2007) in which the liquidity premium is averaged across regimes.
} 


\begin{tabular}{cccccc}
\hline \hline \multirow{2}{*}{ Parameters } & \multicolumn{4}{c}{ the equilibrium discount rates $r_{i}$} \\
\cline { 2 - 6 } & & Single & Bull & bear & Average \\
\hline Baseline Values & & 7.18 & 6.52 & 12.54 & 7.52 \\
\hline \multirow{2}{*}{$\beta=0.02$} & $\times 1.2$ & 7.58 & 6.91 & 12.95 & 7.92 \\
\cline { 2 - 6 } & $\times 0.8$ & 6.78 & 6.12 & 12.12 & 7.11 \\
\hline \multirow{2}{*}{$\beta=0.02, \lambda_{B}=0.42$} & $\times 1.2$ & 7.58 & 6.60 & 12.62 & 7.76 \\
\cline { 2 - 6 } & $\times 0.8$ & 6.78 & 6.46 & 12.47 & 7.28 \\
\hline \hline \multirow{2}{*}{$\beta=0.02, \lambda_{b}=2.112$} & $\times 1.2$ & 7.58 & 7.19 & 13.19 & 8.04 \\
\cline { 2 - 6 } & $\times 0.8$ & 6.78 & 5.74 & 11.78 & 6.94 \\
\hline \hline
\end{tabular}

Table 1: Comparative Statistics. The baseline parameter values are fixed as follows: $\beta=0.02, \gamma=3, \mu_{B}^{D}=0.0218, \sigma_{B}^{D}=0.0117, \mu_{b}^{D}=0.0040, \sigma_{b}^{D}=$ $0.0387, \lambda_{B}=0.42, \lambda_{b}=2.112$. Each row displays the equilibrium discount rates $(\%)$ when parameter values are changed. Each column displays parameter values, single regime case, Bull regime case, bear regime case, and the average across regimes calculated by $\bar{r}=\frac{\lambda_{b}}{\lambda_{B}+\lambda_{b}} r_{B}+\frac{\lambda_{B}}{\lambda_{B}+\lambda_{b}} r_{b}$. For the single regime case, the expected consumption growth rate and volatility of consumption growth rate are set to 0.0182 and 0.0216 , respectively (Yang, 2011). 


\section{References}

Cochrane, J. H. 2017. Macro-Finance. Review of Finance. 21 945-985.

Hamilton, J. D. 1989. A New Approach to the Economic Analysis of Nonstationary Time Series and the Business Cycle. Econometrica. 57 357-384.

Jang, B. -G., H. K. Koo, H. Liu, M. Loewenstein. 2007. Liquidity Premia and Transaction Costs. Journal of Finance. 56 2329-2366. 29 702-730.

Lucas, R. E. 1978. Asset Prices in an Exchange Economy. Econometrica. 46 1429-1445.

Ramsey, F. P. 1928. A Mathematical Theory of Saving. The Economic Journal. 38 543-559.

Yang, W. 2011. Business Cycles and Regime-Shift Risk. Working Paper. 